Haushühnern zusammen im Hofe gehabt, das jedoch durch Zufall getödtet, nicht zum vollen Alter kam. -

Eine andere Mittheilung wurde mir heute gemacht, die gleichfalls interessant und neu ist. Es ist kein Zweifel an der Sache. In Cremorne Garden, eine Meile von Melbourne, ist eine Art See, der in Verbindung mit der Yarra steht. In diesem See baute ein schwarzer Schwan (Cygnus atratus,) sein Nest und vor 14 Tagen begann das Weibchen zu brüten. Hochwasser schwellte die Yarra so sehr, dass die Ufer uberfluthet wurden und der See um mehrere Fuss stieg. Was thun die schönen schwarzen Thiere: das Weibchen verlässt $n i c h t$ das Nest; das Männchen bringt Massen von Schilf, Holz und dergl. dem Weibchen zu, dieses steckt diese Pflanzenreste mit seinem Schnabel unter sich ins Nest, bringt den Kopf dabei oft unter Wasser um tief genug zu reichen und erhöht sich, die Eier und das Nest so hoch, dass es von der Fluth, so weit wie nöthig, verschont blieb. Das Wasser ist jetzt wieder gefallen und man kann das Nest drei Fuss hoch über dem Wasserspiegel hervorragen sehen, und stark genug um die brütende Mutter zu tragen.

\title{
Die geistige Inferiorität des weiblichen Geschlechts bei den Vögeln.
}

Ein Beitrag zur Thierpsychologie.

Von

Pfarrer F. H. Snell.

„Und er soll dein Herr sein" - dieses uralte Wort, welches das Weib dem Manne unterordnete, gilt nicht nur von den Menschen (wiewohl in neuerer Zeit allerlei Emancipationsgelüste dagegen aufgetaucht sind), sondern auch von den Thieren, namentlich auch von den $\mathrm{V}$ ög eln. Es zeigt sich dies auf das Eklatanteste bei dem Nestbau. Der Frühling kommt, die zarten Triebe der Liebe regen sich in der Vogelbrust, und es muss nun vor Allem ein Nest gebaut werden! Aber wo soll dies geschehen? Es gibt der passenden Orte so viele, und es kann doch nur Einer gewählt werden! Es müssen daher die beiden Willen der Gatten sich vereinigen, um eine Wahl zu treffen: wer von ihnen gibt dabei den Ausschlag? Es ist immer das Männchen, welches dies thut. Man beobachte z. B. ein Buchfinkenpaar (Fringilla coelebs). Das Männchen fliegt von Zweig zu Zweig, von Baum zu Baum und probirt überall, wo die Zweige sich theilen, indem es sich zwischen 
dieselben hineinsetzt und sich daselbst wendet und dreht, um durch das Gefühl (oder vielmehr den „Tastsinn “) zu erforschen, ob ein Nest von der erforderlichen Grösse und Rundung anzubringen sei. Das Weibchen folgt nach und setzt sich ebenfalls auf die sondirte Stelle, sobald das Mänuchen dieselbe verlassen hat, um diese nun seinerseits auch zu prüfen. Freilich wäre Letzteres eigentlich ganz überflüssig, da sich das Weibchen doch zuletzt unweigerlich dem definitiven Beschlusse des Männchens unterwirft; aber es gibt dadurch dennoch auf eine schöne Weise zu erkennen, dass seine Unterwerfung nicht auf einem äusseren $\mathrm{Z}$ wange, sondern auf innerer Nothwendigkeit, die ja mit der Freiheit identisch ist, beruht. Das aber ist festzuhalten, dass das Männchen es ist, welches den Nistort bestimmt.

Es ist dieses Beispiel um so schlagender, da grade bei der erwähnten Vogelart das Männchen beim Nestbau nicht mithilft. Dasselbe lässt vielmehr von dem Moment an, wo es über den zu wählenden Nestplatz seine männliche Entscheidung getroffen hat, dem Weibchen vollkommene Freiheit in der Ausführung derselben, fliegt auch nicht mehr voran, sondern folgt nun seinerseits dem Weibchen, wohin sich dieses zum Aufsuchen der passendsten Baumaterialien auch wenden mag, wobei es die kunstfertige Baumeisterin durch seinen Gesang und allerlei verliebte Scherze bei ihrem mühsamen Geschäfte zu erheitern sich bemüht. -

Sehr leicht kann man die in Rede stehende Beobachtung auch bei den $\mathrm{Haustauben}$ machen. Es ist immer der Tauber, welcher den Nestplatz auswählt, und dessen eigenthümlich lockendem Zurufe die Täubin stets folgt. Es ist dies sogar dann der Fall, wenn der Tauber, durch besondere Hindernisse veranlasst, einen total unzweckmässigen und unbrauchbaren Platz aussucht. So besass ich einmal einen Tauber, der durch einen Einbruch des Marders in meinen Schlag so scheu geworden war, dass er sich weder zur Rückkehr in den Schlag bewegen, noch auch einfangen liess, sondern sein Nest auf den nahen Kirchthurm baute. Ich verscheuchte ihn von da durch abendlichen Lärm und nahm die Eier weg. Er zog sich nun wieder in die Nähe des Schlages, in welchen die übrigen Tauben längst wieder eingegangen waren, getraute sich aber nicht, hineinzugehen, sondern trieb sich vor demselben herum, und wählte einen Nistplatz auf einem schiefen Vorsprung des Daches an der Giebelseite des Hauses. Die Täubin folgte dem Rufe des Taubers, obgleich auf diesem Nistplatze wegen seiner schiefen Neigung nicht ein einziges Reischen, geschweige denn Eier hätten liegen bleiben künnen. - Der Tauber erkannte dies auch bald selbst, gab 
den dummen Plan auf und wagte sich nun allmählich in die Fluglöcher hinein bis auf das innere Sitzbrett, welches an denselben angebracht war; aber nicht weiter. Auf diesem schmalen Brett vielmehr stimmte er nun seinen Nestruf an, und die Täubin folgte abermals unweigerlich, obgleich dieser Ort nicht viel passender war, als der andern, da die übrigen Bewohner des Schlages bei ihrem Aus- und Eingehen beständig über das Brett schritten und die zusammengetragenen Reiser herunterwarfen. Endlich sah der Tauber, der sich mittlerweile auch wieder mehr an den Schlag gewöhnt hatte, doch die Vergeblichkeit seines Nestbauens ein und okkupirte nun ein geflochtenes Nest im Innern des Schlages. Niemand mochte sich wohl darüber mehr freuen, als seine Täubin; aber sie selbst hatte nie den geringsten Versuch gemacht, eine solche Aenderung herbeizuführen.

Selbst bei dem Haushuhn, welches doch durch die Zähmung seinem Naturzustande in weit höherem Grade entfremdet ist, als die Taube, und welches in Polygamie lebt, finden sich noch unverkennbare Andeutungen dieses Verhältnisses. Der Hahn weist sehr häufig in Ställen und Scheunen dem Huhne den Platz an, wohin es seine Eier legen soll. Er setzt sich nämlich mit einem eigenthümlichen Locken auf den auserwählten Nestplatz und das Huhn vor ihn. Dann steht ersterer auf und das letztere nimmt den Platz ein, um nun seine Eier dahin regelmässig zu legen.

Dieses Verhältniss der Unterordnung des weiblichen Geschlechts unter das männliche wird sich wohl in der Fortpflanzungsgeschichte aller Vögel beobachten lassen. Scheinbare Ausnahmen kommen allerdings bei solchen Vögeln vor, deren natürliche Brutörter von ganz besonderer Beschaffenheit, daher oft ein Gegenstand des Streites zwischen verschiedenen Paaren, überhaupt nicht überall zu finden sind, wie z. B. bei den Höhlenbrütern. Nicht minder ist dies der Fall bei denjenigen Arten, welche ein und dasselbe Nest, wenn sie es einmal gebaut haben, nun immer wieder und viele Jahre hinter einander beziehen, oder welche sich gar fremder, herrenloser, oder gewaltsam ihrem Eigenthümer entrissener Nester bedienen und nur in Ermangelung solcher ein eigenes zu bauen sich die Mühe nehmen. Bei diesen Vögeln geschieht es nämlich sehr häufig, dass, wenn das Männchen weggeschossen wird oder sonst verunglückt, sich ein neuer Gatte zu dem verwittweten Weibchen gesellt und zugleich auch dasselbe Nest in Besitz nimmt. So schrieb mir vor kurzem ein Freund *) Folgendes über den Falco tinnunculus:

*) Herr Oekonom Unzicker, (dermalen zu Hof Vetzberg bei Giessen), Verfasser mehrerer werthvollen ornithologischen Beiträge in den "Jahrbüchern des Vereins für Naturkunde im Ilerzogthum Nassau." 
„Ich schoss bei einem Horste desselben, welcher in einer weiten Höhlung einer Eiche angebracht war, das Männchen für meine Sammlung. Zwei Tage später ging ich wieder hin, um der trauernden Wittwe einen Besuch zu machen, fand aber zu meiner Verwunderung, dass sie sich schon wieder vermählt hatte, indem Männchen und Weibchen kreischend den Baum umkreisten, wie früher." - Solche Ausnahmen von der Regel sind aber, wie bemerkt, vielleicht nur scheinbar. Denn (um bei dem eben angeführten speciellen Falle zu bleiben) solche weite und nicht tiefe Baumhöhlen, wie sie der Thurmfalke braucht, finden sich nicht überall. Wenn also ein unbeweibtes Mànnchen, welches, eine Gattin suchend, umherstreift, mit dieser zugleich auch einen bequemen Nistort findet, wie vielleicht in der ganzen Gegend keiner mehr anzutreffen ist, so wird es diesen gewiss nicht muthwilliger Weise, am wenigsten aber etwa blos um seine männliche Autorität zu behaupten, wieder aufgeben und nun aufs Gerathewohl nach einem andern suchen. Vielmehr ist wohl anzunehmen, dass das Männchen einen solchen passenden Brüteplatz selbst dann okkupirt haben würde, wenn das aufgefundene Weibchen auch nicht schon Besitzerin desselben gewesen wäre und dass demselben nur das Entdecken der Höhle durch das Weibchen erleichtert wurde. So ists wohl bei allen Raubvögeln, die ja überhaupt nicht gern einen neuen Horst anlegen, sondern immer lieber sich mit dem blosen Ausbessern der alten begnügen, selbst wenn diese ursprünglich von Raben u. dergl. Vögeln gebaut worden sind.

Dies ist meine unmaassgebliche Ansicht von der Sache.

Viel merkwürdiger freilich wäre es, wenn diese meine Ansicht falsch, wenn bei den Raubvögeln die Ausnahme von der Regel nicht blos eine scheinbare, sondern eine wirkliche wäre, wenn also bei diesen das Weibchen in der fraglichen Hinsicht nisht umsonst seine bedeutendere Körpergrösse und seine „Hosen" hätte, (wiewohl es letztere mit dem Männchen theilt), sondern wirklich auch geistig dem Männchen uberlegen wäre und diese Superiorität bei der Auswahl des Nistortes bethätigte. Es liesse sich hierfür auch noch geltend machen, dass z. B. bei Falco Nisus das Weibchen offenbar mehr Muth und Kühnheit besitzt, als das Männchen, indem ersteres z. B. ganz häufig die Spatzen aus den Höfen holt, wäbrend letzteres nur äusserst selten oder fast nie sich so weit wagt *). Auch bei Falco palumbarius habe ich, so oft

-) Seit zwei Jahren habe ich ganz speciell meine Beobachtungen darauf gerichtet, aber wohl unzähligemal Sperberweibchen, nicht ein einzigesmal aber ein Männchen im biesigen Dorfe hinter den Sperlingen gesehen. Nur das Mánnchen ist ein "Finkenhabicht", das Weibchen eher ein "Spatzenhabicht." 
und geflissentlich ich den Vogel auch beobachtet habe, an dem Weibchen niemals einen Mangel an Entschlossenheit und Verwegenheit im Vergleiche mit dem Männchen gefunden, wiewohl andere Beobachter dies behaupten.

Doch es kommt schliesslich auf die Beobachtung an, wer von den beiden Gatten bei den Raubvögeln die Wahl des Nestplatzes bestimmt. Die meisten derselben sind freilich wegen ihrer Scheu nicht leicht bei diesem Geschäfte zu beobachten. Leicht aber muss dies sein bei Falco tinnunculus, da, wo er in der Nähe der Menschen auf Thürmen oder Ruinen nistet. Ich habe niemals Gelegenheit gehabt, ihn so zu beobachten.

Hohenstein in Nassau, im Juli 1858.

Das Vorkommen von Tichodroma phoenioopterre und Lestris parasitica bel Lyon. - Mehrere Naturforscher haben das Vorkommen des rothflügeligen Mauerlaufers in den Umgebungen von Lyon in Zweifel gezogen; selbst mein gelehrter Freund, Herr Léon 0lph. Galliard hat ihn in seinem Kataloge nur mit einem Fragezeichen aufgeführt. Obgleich nun eine Wohnortsfrage wohl nicht von ganz besonderer Bedeutung ist, so erlaube ich mir dennoch einige Beobachtungen zu geben, die ich über diesen Gegenstand gemacht habe.

Ich selbst sah diesen Mauerläufer mehrmals, besonders in der Richtung des Departement de l'Ain, auf der Mauer der Eisenbahn gegen les Balmes de la Pape. Unter anderm sah ich ihn einmal mit Herrn Lembert, einem meiner Freunde, der ebenfalls Naturforscher ist, und der ihn zuerst bemerkte. Mehrere Jäger haben ihn in der Nähe von Lyon geschossen, und Herr Merlin, ein junger Mann, der bereits eine hübsche Sammlung von Vögeln Frankreichs zusammengebracht hat, besitzt einen, der, wie er sagt, zu Genas, einem Dorfe 3 Stunden von Lyon, geschossen wurde. Man kann also diese Vogel-Art unserer Gegend nicht streitig machen, obwohl sie daselbst selten zu nennen ist. Temminck, in seinem vortrefflichen Handbuche der Ornithologie, giebt für den Vogel folgende Wohnorte an: „Die mittäglichen Länder, sehr häufig auf den Schweizeralpen, in Spanien, Italien; stets auf den höchsten Bergen, selten auf Bergen mittlerer Höhe, niemals im Norden." Wie man sieht, spricht er gar nicht von Frankreich, wo diese Art auf den Briançoner-Alpen doch so gewöhnlich ist. Ich sah' sie öfter schiessen und, wie ich oben bemerkte, findet man sie selbst in der Umgegend von Balmes - schroffabschüssiger Ausläufer einer Hügelreihe - , das doch lange nicht die Höhe mittlerer Berge erreicht! Einem Ornithologen 


\section{$2 \mathrm{BHL}$ Biodiversity Heritage Library}

1858. "Die geistige Inferiorität des weiblichen Geschlechts bei den Vögeln." Journal fu

r Ornithologie 6, 394-398. https://doi.org/10.1007/bf02010066.

View This Item Online: $\underline{\text { https://www.biodiversitylibrary.org/item/49379 }}$

DOI: https://doi.org/10.1007/bf02010066

Permalink: https://www.biodiversitylibrary.org/partpdf/141371

\section{Holding Institution}

Natural History Museum Library, London

\section{Sponsored by}

Natural History Museum Library, London

\section{Copyright \& Reuse}

Copyright Status: Public domain. The BHL considers that this work is no longer under copyright protection.

This document was created from content at the Biodiversity Heritage Library, the world's largest open access digital library for biodiversity literature and archives. Visit BHL at https://www.biodiversitylibrary.org. 